\title{
Flat space holography and the complex Sachdev-Ye-Kitaev model
}

\author{
Hamid Afshar@, ${ }^{1,2, *}$ Hernán A. González ${ }^{3, \dagger}$ Daniel Grumiller ${ }^{1,+}$ and Dmitri Vassilevich ${ }^{4,5,8}$ \\ ${ }^{1}$ Institute for Theoretical Physics, TU Wien, Wiedner Hauptstr. 8, A-1040 Vienna, Austria \\ ${ }^{2}$ School of Physics, Institute for Research in Fundamental Sciences (IPM), \\ P.O. Box 19395-5531 Tehran, Iran \\ ${ }^{3}$ Facultad de Artes Liberales, Universidad Adolfo Ibáñez, \\ Diagonal Las Torres 2640 Peñalolén, Santiago, Chile \\ ${ }^{4}$ CMCC, Universidade Federal do ABC, Santo Andre CEP 09210-80, Sao Paulo, Brazil \\ ${ }^{5}$ Physics Department, Tomsk State University, Tomsk 634050, Russia
}

(Received 25 November 2019; accepted 27 March 2020; published 24 April 2020)

\begin{abstract}
We provide the first steps toward a flat space holographic correspondence in two bulk spacetime dimensions. The gravity side is described by a conformally transformed version of the matterless CallanGiddings-Harvey-Strominger model. The field theory side follows from the complex Sachdev-Ye-Kitaev model in the limit of large specific heat and vanishing compressibility. We derive the boundary action analogous to the Schwarzian as the key link between gravity and field theory sides and show that it coincides with a geometric action discovered recently by one of us [H. R. Afshar, Warped Schwarzian theory, J. High Energy Phys. 02 (2020) 126].
\end{abstract}

DOI: 10.1103/PhysRevD.101.086024

\section{INTRODUCTION}

The Sachdev-Ye-Kitaev (SYK) model [1-3] reinvigorated studies of Jackiw-Teitelboim (JT) gravity [4,5] since in a certain limit it is the gravity dual of the former [6]. This holographic relationship, dubbed $\mathrm{nAdS}_{2} / \mathrm{nCFT}_{1}$, inspired numerous research activities in the past few years in gravity and condensed-matter communities; see e.g., [7-46]. One crucial piece of the puzzle is the Schwarzian action $[1,6]$ that arises in the large $N$ and strong coupling limit on the quantum mechanics side and, upon imposing suitable boundary conditions, also on the gravity side.

Given the impressive evidence for AdS/CFT realizations of holography, it is justified to ask how general the holographic principle works, if it works beyond the AdS/CFT correspondence and in particular if and how it works in asymptotically flat spacetimes. See [47-70] and references therein for selected earlier results in flat space holography.

The main goal of our paper is to find a model analogous to JT that leads to a holographic relationship involving flat

\footnotetext{
*afshar@hep.itp.tuwien.ac.at

†hernan.gonzalez@uai.cl

*grumil@hep.itp.tuwien.ac.at

dvassil@gmail.com
}

Published by the American Physical Society under the terms of the Creative Commons Attribution 4.0 International license. Further distribution of this work must maintain attribution to the author(s) and the published article's title, journal citation, and DOI. Funded by SCOAP ${ }^{3}$. space instead of $\mathrm{AdS}_{2}$, with some suitable replacement of the Schwarzian action.

The Calla-Giddings-Harvey-Strominger (CGHS) model [71] is a prime candidate for the gravity side of flat space holography since all solutions have asymptotically vanishing Ricci scalar. This opens up the prospect to construct a concrete holographic correspondence between flat space dilaton gravity in $1+1$ dimensions and some cleverly designed quantum system of (complex) fermions in $0+1$ dimensions.

The principal result of this paper is that the flat space analog of the Schwarzian action is given by

$I^{\mathrm{tw}}[h, g]=\kappa \int_{0}^{\beta} \mathrm{d} \tau\left(\mathcal{T} h^{\prime 2}-g^{\prime}\left(i \mathcal{P} h^{\prime}+\frac{h^{\prime \prime}}{h^{\prime}}\right)+g^{\prime \prime}\right)$,

where $\kappa$ is a coupling constant, $\beta$ is inverse temperature, and prime denotes derivative with respect to $\tau$, the time direction along the boundary. The time-reparametrization field $h(\tau+\beta)=h(\tau)+\beta$ is quasiperiodic and the phase field $g(\tau)$, in the absence of winding, is periodic. When the functions $\mathcal{T}$ and $\mathcal{P}$ are constant, we refer to them as mass and charge, respectively. While mass can be arbitrary, it will turn out that regularity demands a linear relationship between charge and temperature. The superscript tw stands for "twisted warped" and stems from the symmetries (7) that govern our action. On the gravity side $\kappa$ is essentially the inverse Newton constant, as evident from our starting point (2). On the field theory side, $\kappa$ is essentially the geometric mean of specific heat at constant charge and zero 
temperature compressibility, as evident from our final equation (32).

The remainder of our paper is organized as follows. We start by gaining some intuition about our gravity model in the metric formulation and then switch to a gauge theoretic formulation. The latter is employed to derive our main result (1). Finally, we recover the boundary action (1) from a scaling limit of complex SYK.

\section{METRIC FORMULATION OF CGHS}

Following Cangemi and Jackiw [72], we manipulate the CGHS action [71] in three ways: (i) for simplicity, we set all matter fields to zero, (ii) we perform a Weyl rescaling (depending on the dilaton $X$ ) of the metric $g_{\mu \nu}$, and (iiii) we "integrate in" an Abelian gauge field $A_{\mu}$ and an auxiliary scalar field $Y$ that is constant on shell. The action $\left(\varepsilon^{\mu \nu}\right.$ is the $\varepsilon$-tensor)

$$
I \widehat{\mathrm{CGHS}}=\frac{\kappa}{2} \int \mathrm{d}^{2} x \sqrt{-g}\left(X R-2 Y+2 Y \varepsilon^{\mu \nu} \partial_{\mu} A_{\nu}\right)
$$

provides a reformulation of the CGHS model referred to as $\widehat{\mathrm{CGH}} \mathrm{S}$. We solve now the $\widehat{\mathrm{CGH} S}$ field equations,

$$
\begin{gathered}
\mathrm{R}=0 \\
\varepsilon^{\mu \nu} \partial_{\mu} A_{\nu}=1, \\
\nabla_{\mu} \nabla_{\nu} X-g_{\mu \nu} \nabla^{2} X=g_{\mu \nu} Y \quad Y=\Lambda=\mathrm{const},
\end{gathered}
$$

with suitable boundary and gauge fixing conditions.

In Eddington-Finkelstein gauge, the most general solution to the Ricci-flatness condition (3) is given by Rindler-type black hole metrics of the form

$$
\mathrm{d} s^{2}=-2 \mathrm{~d} u \mathrm{~d} r+2(\mathcal{P}(u) r+\mathcal{T}(u)) \mathrm{d} u^{2} .
$$

The loosest set of boundary conditions compatible with the gauge fixing (6) allows fluctuations of both free functions, $\delta \mathcal{P} \neq 0 \neq \delta \mathcal{T}$. These boundary- and gaugefixing conditions are preserved by asymptotic Killing vectors $\quad \xi(\varepsilon, \eta)=\varepsilon(u) \partial_{u}-\left(\varepsilon^{\prime}(u) r+\eta(u)\right) \partial_{r} \quad$ since $\mathcal{L}_{\xi} g_{\mu \nu}=\mathcal{O}\left(\delta g_{\mu \nu}\right), \quad$ namely, $\quad \mathcal{L}_{\xi} g_{r r}=0=\mathcal{L}_{\xi} g_{u r} \quad$ and $\mathcal{L}_{\xi} g_{u u}=\delta_{\xi} \mathcal{P} r+\delta_{\xi} \mathcal{T}$, with $\delta_{\xi} \mathcal{P}=\varepsilon \mathcal{P}^{\prime}+\varepsilon^{\prime} \mathcal{P}+\varepsilon^{\prime \prime} \quad$ and $\delta_{\xi} \mathcal{T}=\varepsilon \mathcal{T}^{\prime}+2 \varepsilon^{\prime} \mathcal{T}+\eta^{\prime}-\eta \mathcal{P}$. Prime is the derivative along retarded time $u$ and $\mathcal{L}_{\xi}$ the Lie-derivative along $\xi$.

The Lie-bracket algebra of the asymptotic Killing vectors $\left[\xi\left(\varepsilon_{1}, \eta_{1}\right), \xi\left(\varepsilon_{2}, \eta_{2}\right)\right]_{\text {Lie }}=\xi\left(\varepsilon_{1} \varepsilon_{2}^{\prime}-\varepsilon_{2} \varepsilon_{1}^{\prime},\left(\varepsilon_{1} \eta_{2}-\right.\right.$ $\left.\left.\varepsilon_{2} \eta_{1}\right)^{\prime}\right)$ in terms of Laurent modes, $L_{n}=\xi\left(\varepsilon=-u^{n+1}, \eta=\right.$ $0)$ and $M_{n}=\xi\left(\varepsilon=0, \eta=u^{n-1}\right)$, yields $\left[L_{n}, L_{m}\right]_{\text {Lie }}=$ $(n-m) L_{n+m}, \quad\left[L_{n}, M_{m}\right]_{\text {Lie }}=-(n+m) M_{n+m}, \quad$ and $\left[M_{n}, M_{m}\right]_{\text {Lie }}=0$. This algebra consists of a Witt subalgebra generated by $L_{n}$ and spin-0 supertranslations generated by $M_{n}$. We refer to it as $\mathrm{BMS}_{2}$ [73].
In axial gauge for the $U(1)$ connection, the field equation (4) is solved by the two-dimensional Coulomb connection $A=r \mathrm{~d} u$. Its preservation under combined diffeomorphisms and gauge transformations, $\delta_{\xi, \sigma} A_{\nu}=$ $\xi^{\mu} \partial_{\mu} A_{\nu}+A_{\mu} \partial_{\nu} \xi^{\mu}+\partial_{\nu} \sigma$, relates the functions $\eta$ and $\sigma$, $\eta=\sigma^{\prime}$, which can be interpreted in two different ways. Either one concludes that $\sigma$ has to contain a $\ln u$ term, since $M_{0}$ is allowed to be nonzero, or one concludes that $M_{0}$ is forbidden, since $\sigma$ is assumed to have a Laurent series around $u=0$. The first option leads to the $\mathrm{BMS}_{2}$ symmetries discussed above. The second option leads to a slight modification of the transformation properties,

$$
\begin{gathered}
\delta_{\xi} \mathcal{P}=\varepsilon \mathcal{P}^{\prime}+\varepsilon^{\prime} \mathcal{P}+\varepsilon^{\prime \prime}, \\
\delta_{\xi} \mathcal{T}=\varepsilon \mathcal{T}^{\prime}+2 \varepsilon^{\prime} \mathcal{T}+\sigma^{\prime \prime}-\sigma^{\prime} \mathcal{P} .
\end{gathered}
$$

In the present paper, we focus on the second option, since it guarantees that the Wilson loop in the complex $u$ plane encircling the origin $u=0$ is gauge invariant, $\delta_{\sigma} \oint A=0$; in other words, there are no winding modes.

Defining instead of $M_{n}$ new Fourier modes $J_{n}$ := $\xi\left(0, \sigma=u^{n}\right)$ yield the asymptotic symmetry algebra in terms of asymptotic Killing vector modes $\left[L_{n}, L_{m}\right]_{\text {Lie }}=$ $(n-m) L_{n+m},\left[L_{n}, J_{m}\right]_{\mathrm{Lie}}=-m J_{n+m}$, and $\left[J_{n}, J_{m}\right]_{\mathrm{Lie}}=0$, which is known as "warped Witt algebra," the centerless version of either the warped conformal algebra [76] or the twisted warped conformal algebra [77].

Finally, the $r r$ component of the field equation (5) is solved by dilaton fields linear in the radial coordinate

$$
X=x_{1}(u) r+x_{0}(u) .
$$

The remaining components of the field equation (5), which involve the functions $x_{i}(u)$, will be determined in the gauge

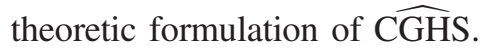

\section{GAUGE THEORY FORMULATION OF CGHS}

For the gauge theoretic formulation as non-Abelian $\mathrm{BF}$ theory $[72,78]$, we use conventions analogous to [79]. The first order form of the C $\widehat{\mathrm{GH}} \mathrm{S}$ bulk action (2) is given by

$$
I^{\mathrm{BF}}[B, \mathcal{A}]=\kappa \int\langle B, F\rangle,
$$

where $\kappa$ is the coupling constant, $B$ is a scalar, and $F=\mathrm{d} \mathcal{A}+\mathcal{A} \wedge \mathcal{A}$ the non-Abelian field strength. The connection

$$
\mathcal{A}=\omega J+e^{a} P_{a}+A Z
$$

contains dualized spin-connection $\omega$, zweibein $e^{a}$, and $U(1)$ connection $A$. The generators obey the Maxwell algebra [80] whose nonzero commutators read $\left[P_{a}, P_{b}\right]=$ $\epsilon_{a b} Z$ and $\left[P_{a}, J\right]=\epsilon_{a}{ }^{b} P_{b}$. The scalar field 


$$
B=X Z+X^{a} \epsilon_{a}^{b} P_{b}+Y J
$$

comprises the dilaton $X$, Lagrange multipliers $X^{a}$ for torsion constraints, and the auxiliary field $Y$. Finally, $\langle$, denotes the bilinear form with nonvanishing entries,

$$
\langle J, Z\rangle=-1\left\langle P_{a}, P_{b}\right\rangle=\eta_{a b}
$$

We use light cone gauge for the Minkowski metric, $\eta_{+-}=1$, in terms of which the Levi-Civitá symbol is $\epsilon_{ \pm}{ }^{ \pm}= \pm 1$, and the gauge algebra reads $\left[P_{+}, P_{-}\right]=Z$ and $\left[P_{ \pm}, J\right]= \pm P_{ \pm}$. Integrating out the Lagrange multipliers $X^{a}$ and solving the torsion constraints, the action (9) with (10)-(12) can be shown to be equivalent to (2).

Boundary conditions compatible with the ones in the metric formulation are given by

$$
\mathcal{A}=b^{-1}(\mathrm{~d}+a) b \quad B=b^{-1} x b,
$$

with $b=\exp \left(-r P_{+}\right)$and

$$
\begin{gathered}
a=\left(\mathcal{T}(u) P_{+}+P_{-}+\mathcal{P}(u) J\right) \mathrm{d} u, \\
x=x^{+}(u) P_{+}+x_{1}(u) P_{-}+Y J+x_{0}(u) Z,
\end{gathered}
$$

where both functions in the connection are allowed to vary, $\delta \mathcal{T} \neq 0 \neq \delta \mathcal{P}$.

The equations of motion reduce to

$$
\mathrm{d} a+a \wedge a=0=\mathrm{d} x+[a, x] .
$$

The first one is obeyed automatically by our ansatz (14). The second one, which states that $x$ is the stabilizer of $a$, holds provided $\mathrm{Y}=\Lambda=$ const., and the following differential equations are fulfilled: $\left(x^{+}\right)^{\prime}=\mathcal{P} x^{+}-\mathcal{T} Y$, $x_{1}^{\prime}=-\mathcal{P} x_{1}+Y$, and $x_{0}^{\prime}=x^{+}-\mathcal{T} x_{1}$. Using them, $x$ is conveniently parametrized by two functions $x_{1}$ and $x_{0}$,

$x=\left(x_{0}^{\prime}+\mathcal{T} x_{1}\right) P_{+}+x_{1} P_{-}+\left(x_{1}^{\prime}+\mathcal{P} x_{1}\right) J+x_{0} Z$.

\section{ASYMPTOTIC SYMMETRIES}

The boundary condition preserving gauge transformations $\delta_{\lambda} B=[B, \lambda]$ and

$$
\delta_{\lambda} \mathcal{A}=d \lambda+[\mathcal{A}, \lambda] \stackrel{!}{=} \mathcal{O}(\delta \mathcal{A})=\left(\mathcal{O}(r) P_{+}+\mathcal{O}(1) J\right) d u
$$

are generated by gauge parameters $\lambda=b^{-1} \epsilon b$ with

$$
\epsilon=\epsilon^{+}(u) P_{+}+\varepsilon(u) P_{-}+\epsilon^{J}(u) J+\sigma(u) Z .
$$

The absence of the $P_{-}$and $Z$ components on the right-hand side of (18) yields consistency relations between the functions in the gauge parameter (19).

$$
\epsilon^{J}=\varepsilon^{\prime}+\mathcal{P} \varepsilon \quad \epsilon^{+}=\sigma^{\prime}+\mathcal{T} \varepsilon .
$$

The boundary condition preserving gauge transformations (18)-(20) imply precisely the transformation laws (7), which is the twisted warped conformal transformation behavior introduced in [77]. Therefore, the analog of the conformal symmetries that govern the Schwarzian action are twisted warped conformal symmetries, which govern our boundary action (1).

\section{BOUNDARY ACTIONS}

Our derivation of the boundary action for C $\widehat{\mathrm{GH}} \mathrm{S}$ follows closely the derivation of the Schwarzian action in Sec. 3 of [79].

From now on, we work in Euclidean signature with periodic boundary time, $t \sim t+\beta$, where $\beta$ is inverse temperature. Mapping Lorentzian to Euclidean results requires the following replacements: $u \rightarrow i u=t, \eta_{a b} \rightarrow$ $\delta_{a b}, P_{ \pm}=P_{1}^{\mathrm{E}} \pm i P_{0}^{\mathrm{E}}, Z=i Z^{\mathrm{E}}, J=i J^{\mathrm{E}}$, and $x_{0,1}=-i x_{0,1}^{\mathrm{E}}$ (with real $x_{0,1}^{\mathrm{E}}$ ). The fields are given by (10) and (11), with all quantities replaced by their Euclidean counterparts. We use the definition $\oint \mathrm{d} t=\int_{0}^{\beta} \mathrm{d} t$ and $\operatorname{dot}$ means $\frac{\mathrm{d}}{\mathrm{d} t}$.

The variation of the BF action (9) apart from the bulk equations of motion yields a boundary term [79],

$$
\delta I^{\mathrm{tw}}=-\kappa \oint \mathrm{d} t\left\langle x, \delta a_{t}\right\rangle .
$$

Our aim is to cancel this boundary term by variation of a boundary action. To this end, we use a convenient representation of the connection, $a_{t}=f_{t} x+G^{-1} \partial_{t} G$. A consistent choice is $G=\exp \left(x_{0}^{\mathrm{E}}\left(i P_{1}^{\mathrm{E}}-P_{0}^{\mathrm{E}}\right)\right) \exp \left(-i \ln \left(-i x_{1}^{\mathrm{E}}\right) J^{\mathrm{E}}\right)$ $\exp \left(-\int^{t} x_{0}^{\mathrm{E}} / x_{1}^{\mathrm{E}} Z^{\mathrm{E}}\right)$ and $f_{t}=1 / x_{1}^{\mathrm{E}}$. We impose as integrability condition that the function $f_{t}$ has a fixed zero mode (which we set to unity with no loss of generality). As shown below, this guarantees that the first variation of the full action vanishes for all variations preserving our boundary conditions.

The variation of the boundary action (21) expands as

$$
\begin{aligned}
\delta I^{\mathrm{tw}}= & -\kappa \oint \mathrm{d} t\left[\delta\left(f_{t} C\right)+C \delta f_{t}\right. \\
& \left.-\left\langle\left(\partial_{t} x+\left[G^{-1} \partial_{t} G, x\right]\right), G^{-1} \delta G\right\rangle+\partial_{t}\left\langle x, G^{-1} \delta G\right\rangle\right],
\end{aligned}
$$

with the bilinear Casimir [83]

$$
C=\frac{1}{2}\langle B, B\rangle=\frac{1}{2}\langle x, x\rangle .
$$

The second term in (22) vanishes on shell since $C$ is constant and $f_{t}$ has a fixed zero mode, while the terms in 
the second line vanish on shell or because they integrate to zero; thus, only the first term remains, which is integrable in field space, leading to the boundary action

$$
I^{\mathrm{tw}}=-\kappa \oint \mathrm{d} t f_{t} C
$$

For future purposes, we define $f_{t}=\dot{f}$, where $f=t+\cdots$ is a quasiperiodic function that has arbitrary Fourier modes but a fixed linear term. The full action

$$
\Gamma^{\mathrm{BF}}[B, \mathcal{A}]=I^{\mathrm{BF}}[B, \mathcal{A}]-\kappa \oint \mathrm{d} t \dot{f} C
$$

has a well-defined variational principle, i.e., its first variation vanishes for all variations that preserve our boundary and on shell conditions (13)-(16).

Plugging the Euclidean version of the expression (17) for $x$ into the bilinear Casimir (23) yields

$$
C=-\frac{1}{(\dot{f})^{2}}\left(\mathcal{T}-\dot{f} x_{0}^{\mathrm{E}} \mathcal{P}+i \dot{f} \dot{x_{0}^{\mathrm{E}}}+i \ddot{f} x_{0}^{\mathrm{E}}\right),
$$

where we used the relation $1 / x_{1}^{\mathrm{E}}=\dot{f}$. Defining additionally $\dot{g}:=i x_{0}^{\mathrm{E}} \dot{f}$, the boundary action (24) with the expression for the Casimir (26) is nearly our final result.

$$
I^{\mathrm{tw}}=\kappa \oint \frac{\mathrm{d} t}{\dot{f}}(\mathcal{T}+i \dot{g} \mathcal{P}+\ddot{g})
$$

The boundary action (27) depends functionally on $f$ and $g$, both of which are boundary scalars, as evident from their transformation behavior under asymptotic symmetries, $\delta_{\lambda} f=\varepsilon \dot{f}$ and $\delta_{\lambda} g=\sigma+\varepsilon \dot{g}$. The latter also shows that $g$ is a phase under $U(1)$ gauge transformations.

As in the JT case [79], we reparametrize the time coordinate along the boundary by a diffeomorphism $\tau:=f(t)$, where $\tau$ is our new (Euclidean) time coordinate with period $\beta$, and introduce the inverse of $f$ as a new field $h(\tau):=-f^{-1}(\tau)$. The other field, $g$, now also depends on $\tau$ and prime from now on means derivative with respect to $\tau$. Implementing this diffeomorphism in the boundary action (27) establishes the boundary action (1) announced in the introduction, where prime means $\frac{\mathrm{d}}{\mathrm{d} \tau}$.

The action (1) is our main result and constitutes the analog of the Schwarzian. Since it has a geometric interpretation as group action for twisted warped coadjoint orbits, governed by the symmetries (7), we refer to it as 'twisted warped action.'. This is analogous to the interpretation of the Schwarzian action as group action for Virasoro coadjoint orbits [86,87]. We refer to [88] and references therein for more on these mathematical aspects.

\section{SOLUTIONS TO TWISTED WARPED THEORY}

We study now classical solutions of the action (1) for constant representatives, $\mathcal{T}=\mathcal{T}_{0}$ and $\mathcal{P}=\mathcal{P}_{0}$. The Hamiltonian formulation involves three canonical pairs $(i=1,2,3)$,

$$
I^{\mathrm{tw}}\left[q_{i}, p_{i}\right]=-\kappa \int_{0}^{\beta} \mathrm{d} \tau\left(p_{i} q_{i}^{\prime}-p_{1} p_{2}-e^{q_{1}} p_{3}\right) .
$$

The relation to the original variables is $q_{3}=\exp \left(i \mathcal{P}_{0} h\right)$, $q_{2}=g+i h \mathcal{T}_{0} / \mathcal{P}_{0}$ while all other canonical variables are of auxiliary nature to get rid of higher derivatives. The interaction term with the exponential in $q_{1}$ also appears in the Schwarzian theory; see Eq. (2.1) in [39]. The key difference is the kinetic term, $p_{1}^{2}$ for the Schwarzian and $p_{1} p_{2}$ for the twisted warped Hamiltonian.

Solving the Hamiltonian equations of motion yields $q_{3}=h_{0}+h_{1} e^{i \tau / \tau_{0}}$ and $q_{2}=g_{0}-i g_{1} \tau+g_{2} e^{i \tau / \tau_{0}}$. These solutions depend on six integration constants, $g_{0}, g_{1}, g_{2}$, $h_{0}, h_{1}, \tau_{0}$, the latter playing the role of the periodicity, $\tau_{0}=\frac{\beta}{2 \pi}$. The integration constants $h_{0}$ and $g_{0}$ are constant shifts, while $h_{1}$ and $g_{2}$ are amplitudes in front of oscillating terms. The remaining constant, $g_{1}$, captures the nonperiodicity of $q_{2}$ and is responsible for the on shell action being nonzero, $\left.I^{\mathrm{tw}}\left[q_{i}, p_{i}\right]\right|_{\mathrm{EOM}}=-2 \pi \kappa g_{1}$.

\section{THERMODYNAMICS}

Assuming $g_{1}$ is independent from temperature allows to deduce the entropy $S=-\left.I^{\mathrm{tw}}\left[q_{i}, p_{i}\right]\right|_{\mathrm{EOM}}=2 \pi \kappa g_{1}$ from the on shell action. Inserting all our definitions we recover the well-known fact that entropy is given by the dilaton at the horizon [89],

$$
S=\left.2 \pi \kappa X\right|_{\text {horizon }}
$$

The result for entropy (29) can be derived along the lines of $[7,43]$. One aspect of this derivation is worth highlighting: the holonomy of $a$ along the thermal cycle must belong to the center of our gauge group for regularity (in order to have contractible thermal cycles). Assuming a single cover, we find that this regularity condition relates to temperature $T=\beta^{-1}$ and charge

$$
\mathcal{P}_{0}=2 \pi T
$$

while mass $\mathcal{T}_{0}$ remains arbitrary. The label "charge" is justified for $\mathcal{P}_{0}$ since the equations of motion imply $\mathcal{P}_{0}=$ $Y$ and $Y$ is the $U(1)$ charge. The label "mass" is justified for $\mathcal{T}_{0}$ as it is the subleading term in the metric (6) and since the associated function $\mathcal{T}$ transforms like a stress tensor (7b) in a twisted warped field theory [77].

A peculiar aspect of CิGHS black hole thermodynamics is that the inverse specific heat (at fixed charge) vanishes, 
$C^{-1}=\left.\frac{1}{T} \mathrm{~d} T\right|_{\delta \mathcal{P}_{0}=0}=0$, since the Hawking-Unruh temperature $T$ trivially does not vary if the charge $\mathcal{P}_{0}$ is kept fixed due to the relation (30). This property is well known [90], but will be crucial for the scaling limit from complex SYK.

\section{SCALING LIMIT FROM COMPLEX SYK}

We turn now to the field theory side, starting with the complex SYK model [6,27,91-93]. The effective action governing the dynamics of the collective low temperature modes of complex SYK is given by (see [27] and Eq. (1.12) in [93])

$$
\begin{aligned}
I^{\mathrm{cSYK}}= & \frac{N K}{2} \int_{0}^{\beta} \mathrm{d} \tau\left(g^{\prime}+\frac{2 \pi i \mathcal{E}}{\beta} h^{\prime}\right)^{2} \\
& -\frac{N \gamma}{4 \pi^{2}} \int_{0}^{\beta} \mathrm{d} \tau\left\{\tan \left(\frac{\pi}{\beta} h\right) ; \tau\right\},
\end{aligned}
$$

where $\{f ; \tau\}:=f^{\prime \prime \prime} / f^{\prime}-\frac{3}{2}\left(f^{\prime \prime} / f^{\prime}\right)^{2}$ is the Schwarzian derivative, $N$ is the (large) number of complex fermions, $N K$ is the zero temperature compressibility, $N \gamma$ is the specific heat at fixed charge, and $\mathcal{E}$ is a spectral asymmetry parameter. The time-reparametrization field $h(\tau+\beta)=$ $h(\tau)+\beta$ is quasiperiodic and the phase field $g(\tau)$, in the absence of winding, is periodic.

According to the thermodynamical discussion above, we are interested in the limit $N \gamma \rightarrow \infty$ in order to obtain our action (1) as limit from the complex SYK effective action (31). This is indeed possible by combining the actions (1) and (31) to the geometric action associated with the twisted warped Virasoro group, known as "warped Schwarzian" $[88], I^{\mathrm{wSch}}=I^{\mathrm{cSYK}}+I^{\mathrm{tw}}$.

Starting from the effective action (31) and shifting $g$ by [88] $g \rightarrow g-\frac{\kappa}{N K}\left(\log h^{\prime}+\frac{2 \pi i}{\beta} h\right)$ yields the action $I^{\text {wSch }}$ with nonvanishing $\kappa$ and shifted specific heat parameter $\hat{\gamma}=\gamma+\frac{36 \pi^{2} \kappa^{2}}{N^{2} K}$. Thus, our boundary action (1) emerges by sending both $\hat{\gamma}$ and $K$ to zero, while keeping fixed $\kappa$. At large $N$, this is achieved by the family of scaling limits,

$$
\gamma=\gamma_{0} N^{a} \quad K=-K_{0} N^{-b} \quad \kappa=\frac{N^{1+\frac{a-b}{2}}}{6 \pi} \sqrt{\gamma_{0} K_{0}} .
$$

The constants $\gamma_{0}$ and $K_{0}$ are independent from $N$ and their product must be positive. The exponents $a>-1, b>1$ lead to infinite specific heat and vanishing zero temperature compressibility, respectively, in the large $N$ limit. Two simple choices are $a=b=2$, leading to $\kappa=\frac{N}{6 \pi} \sqrt{\gamma_{0} K_{0}}$, and $a=0, b=2$, leading to $\kappa=\frac{1}{6 \pi} \sqrt{\gamma_{0} K_{0}}$.

\section{CONCLUSIONS}

We derived on the gravity side the boundary action (1) as a first step toward a two-dimensional model for flat space holography. We showed that the field theory side of our proposal for flat space holography emerges as a triple scaling limit of complex SYK: large $N$, large coupling (or small temperature) and large specific heat, while keeping fixed (with an adjustable scaling in $N$ ) the geometric mean of specific heat and zero temperature compressibility. As evident from (32), this geometric mean (up to a factor $N^{1+\frac{a-b}{2}} /(6 \pi)$ ) is the coupling constant $\kappa$ in (1).

Starting from our flat space holographic description, numerous further research avenues can now be pursued, inspired by corresponding SYK-related results or by generic aspects of two-dimensional dilaton gravity (see [94] for a review and [95] for a list of models). Not intending to do justice to the vast literature on these subjects we highlight just one intriguing aspect, namely, the role of chaos in flat space holography. By analogy to the $\mathrm{AdS}_{2}$ case $[11,96]$, we expect saturation of the chaos bound, i.e., a Lyapunov exponent given by $\lambda_{L}=2 \pi T$. It should be rewarding to verify this through explicit calculations.

\section{ACKNOWLEDGMENTS}

We thank Arjun Bagchi, Bob McNees, Stefan Prohazka, Jakob Salzer, and Carlos Valcárcel for collaborations on related subjects and for discussions. D. G. is particularly grateful to Jakob Salzer for joint unpublished work on $\mathrm{BMS}_{2}$ in two-dimensional dilaton gravity. This work was supported by the Austrian Science Fund (FWF), Projects No. P 28751, No.P30822, and No.P32581.D. V. was supported in parts by the São Paulo Research Foundation (FAPESP), Project No. 2016/03319-6, by Grant No. 303807/2016-4 of CNPq, by the RFBR Project No. 18-02-00149-a, and by the Tomsk State University Competitiveness Improvement Program. H. G. is funded by Fondecyt Grant No. 11190427. H. A. and D. G. acknowledge the Iran-Austria IMPULSE project grant supported and run by Kharazmi University and OeAD-GmbH. 
[1] A. Kitaev, A simple model of quantum holography, KITP Strings Seminars (2015), http://online.kitp.ucsb.edu/online/ entangled15/ and http://online.kitp.ucsb.edu/online/ entangled15/kitaev2/.

[2] S. Sachdev and J. Ye, Gapless Spin Fluid Ground State in a Random, Quantum Heisenberg Magnet, Phys. Rev. Lett. 70, 3339 (1993).

[3] S. Sachdev, Holographic Metals and the Fractionalized Fermi Liquid, Phys. Rev. Lett. 105, 151602 (2010).

[4] R. Jackiw, Liouville field theory: A two-dimensional model for gravity? in Quantum Theory Of Gravity, edited by S. Christensen (Adam Hilger, Bristol, 1984), pp. 403-420.

[5] C. Teitelboim, The Hamiltonian structure of twodimensional space-time and its relation with the conformal anomaly, in Quantum Theory Of Gravity, edited by S. Christensen (Adam Hilger, Bristol, 1984), pp. 327-344.

[6] J. Maldacena and D. Stanford, Remarks on the SachdevYe-Kitaev model, Phys. Rev. D 94, 106002 (2016).

[7] D. Grumiller, J. Salzer, and D. Vassilevich, $\mathrm{AdS}_{2}$ holography is (non-)trivial for (non-)constant dilaton, J. High Energy Phys. 12 (2015) 015.

[8] J. Polchinski and V. Rosenhaus, The spectrum in the Sachdev-Ye-Kitaev model, J. High Energy Phys. 04 (2016) 001.

[9] Y.-Z. You, A. W. W. Ludwig, and C. Xu, Sachdev-YeKitaev model and thermalization on the boundary of many-body localized Fermionic symmetry protected topological states, Phys. Rev. B 95, 115150 (2017).

[10] A. Jevicki, K. Suzuki, and J. Yoon, Bi-local holography in the SYK model, J. High Energy Phys. 07 (2016) 007.

[11] K. Jensen, Chaos in $\mathrm{AdS}_{2}$ Holography, Phys. Rev. Lett. 117, 111601 (2016).

[12] J. Maldacena, D. Stanford, and Z. Yang, Conformal symmetry and its breaking in two dimensional nearly antide-Sitter space, Prog. Theor. Exp. Phys. 2016, 12 C104 (2016).

[13] J. Engelsöy, T. G. Mertens, and H. Verlinde, An investigation of $\mathrm{AdS}_{2}$ backreaction and holography, J. High Energy Phys. 07 (2016) 139.

[14] D. Bagrets, A. Altland, and A. Kamenev, SachdevYeKitaev model as Liouville quantum mechanics, Nucl. Phys. B911, 191 (2016).

[15] L. García-Álvarez, I. L. Egusquiza, L. Lamata, A. del Campo, J. Sonner, and E. Solano, Digital Quantum Simulation of Minimal AdS/CFT, Phys. Rev. Lett. 119, 040501 (2017).

[16] M. Cvetič and I. Papadimitriou, $\mathrm{AdS}_{2}$ holographic dictionary, J. High Energy Phys. 12 (2016) 8; J. High Energy Phys. 01 (2017) 120.

[17] A. Jevicki and K. Suzuki, Bi-local holography in the SYK model: Perturbations, J. High Energy Phys. 11 (2016) 046.

[18] Y. Gu, X.-L. Qi, and D. Stanford, Local criticality, diffusion and chaos in generalized Sachdev-Ye-Kitaev models, J. High Energy Phys. 05 (2017) 125.

[19] D. J. Gross and V. Rosenhaus, A generalization of SachdevYe-Kitaev, J. High Energy Phys. 02 (2017) 093.

[20] M. Berkooz, P. Narayan, M. Rozali, and J. Simón, Higher dimensional generalizations of the SYK Model, J. High Energy Phys. 01 (2017) 138.
[21] A. M. García-García and J. J. M. Verbaarschot, Spectral and thermodynamic properties of the Sachdev-Ye-Kitaev model, Phys. Rev. D 94, 126010 (2016).

[22] S. Banerjee and E. Altman, Solvable model for a dynamical quantum phase transition from fast to slow scrambling, Phys. Rev. B 95, 134302 (2017).

[23] W. Fu, D. Gaiotto, J. Maldacena, and S. Sachdev, Supersymmetric Sachdev-Ye-Kitaev models, Phys. Rev. D 95, 026009 (2017); Phys. Rev. D 95, 069904(A) (2017).

[24] E. Witten, An SYK-like model without disorder, J. Phys. A 52, 474002 (2019).

[25] J. S. Cotler, G. Gur-Ari, M. Hanada, J. Polchinski, P. Saad, S. H. Shenker, D. Stanford, A. Streicher, and M. Tezuka, Black holes and random matrices, J. High Energy Phys. 05 (2017) 118.

[26] I. R. Klebanov and G. Tarnopolsky, Uncolored random tensors, melon diagrams, and the Sachdev-Ye-Kitaev models, Phys. Rev. D 95, 046004 (2017).

[27] R. A. Davison, W. Fu, A. Georges, Y. Gu, K. Jensen, and S. Sachdev, Thermoelectric transport in disordered metals without quasiparticles: The Sachdev-Ye-Kitaev models and holography, Phys. Rev. B 95, 155131 (2017).

[28] C. Peng, M. Spradlin, and A. Volovich, A supersymmetric SYK-like tensor model, J. High Energy Phys. 05 (2017) 062.

[29] C. Krishnan, S. Sanyal, and P. N. Bala Subramanian, Quantum chaos and holographic tensor models, J. High Energy Phys. 03 (2017) 056.

[30] G. Turiaci and H. Verlinde, Towards a 2d QFT analog of the SYK model, J. High Energy Phys. 10 (2017) 167.

[31] Z. Bi, C.-M. Jian, Y.-Z. You, K. A. Pawlak, and C. Xu, Instability of the non-Fermi liquid state of the Sachdev-YeKitaev Model, Phys. Rev. B 95, 205105 (2017).

[32] T. Li, J. Liu, Y. Xin, and Y. Zhou, Supersymmetric SYK model and random matrix theory, J. High Energy Phys. 06 (2017) 111.

[33] R. Gurau, Quenched equals annealed at leading order in the colored SYK model, Europhys. Lett. 119, 30003 (2017).

[34] G. Mandal, P. Nayak, and S. R. Wadia, Coadjoint orbit action of Virasoro group and two-dimensional quantum gravity dual to SYK/tensor models, J. High Energy Phys. 11 (2017) 046.

[35] D. J. Gross and V. Rosenhaus, The bulk dual of SYK: Cubic couplings, J. High Energy Phys. 05 (2017) 092.

[36] P. Caputa, N. Kundu, M. Miyaji, T. Takayanagi, and K. Watanabe, Anti-de Sitter Space from Optimization of Path Integrals in Conformal Field Theories, Phys. Rev. Lett. 119, 071602 (2017).

[37] S.-K. Jian and H. Yao, Solvable Sachdev-Ye-Kitaev Models in Higher Dimensions: From Diffusion to Many-Body Localization, Phys. Rev. Lett. 119, 206602 (2017).

[38] X.-Y. Song, C.-M. Jian, and L. Balents, A Strongly Correlated Metal Built from Sachdev-Ye-Kitaev Models, Phys. Rev. Lett. 119, 216601 (2017).

[39] T. G. Mertens, G. J. Turiaci, and H. L. Verlinde, Solving the Schwarzian via the conformal bootstrap, J. High Energy Phys. 08 (2017) 136.

[40] C. Krishnan and K. V. P. Kumar, Towards a finite- $N$ hologram, J. High Energy Phys. 10 (2017) 099. 
[41] A. M. García-García, B. Loureiro, A. Romero-Bermúdez, and M. Tezuka, Chaotic-Integrable Transition in the Sachdev-Ye-Kitaev Model, Phys. Rev. Lett. 120, 241603 (2018).

[42] T. Azeyanagi, F. Ferrari, and F. I. Schaposnik Massolo, Phase Diagram of Planar Matrix Quantum Mechanics, Tensor, and Sachdev-Ye-Kitaev Models, Phys. Rev. Lett. 120, 061602 (2018).

[43] D. Grumiller, R. McNees, J. Salzer, C. Valcárcel, and D. Vassilevich, Menagerie of $\mathrm{AdS}_{2}$ boundary conditions, J. High Energy Phys. 10 (2017) 203.

[44] F. M. Haehl and M. Rozali, Fine Grained Chaos in $\mathrm{AdS}_{2}$ Gravity, Phys. Rev. Lett. 120, 121601 (2018).

[45] A. Altland, D. Bagrets, and A. Kamenev, Quantum Criticality of Granular Sachdev-Ye-Kitaev Matter, Phys. Rev. Lett. 123, 106601 (2019).

[46] S. Hirano and Y. Lei, Nearly $\mathrm{AdS}_{2}$ holography in quantum CGHS model, J. High Energy Phys. 01 (2020) 178.

[47] J. Polchinski, S matrices from AdS space-time, arXiv: hep-th/9901076.

[48] L. Susskind, Holography in the flat space limit, AIP Conf. Proc. 493, 98 (1999).

[49] S. B. Giddings, Flat space scattering and bulk locality in the AdS/CFT correspondence, Phys. Rev. D 61, 106008 (2000).

[50] R. B. Mann and D. Marolf, Holographic renormalization of asymptotically flat spacetimes, Classical Quantum Gravity 23, 2927 (2006).

[51] C. Dappiaggi, V. Moretti, and N. Pinamonti, Rigorous steps towards holography in asymptotically flat spacetimes, Rev. Math. Phys. 18, 349 (2006).

[52] G. Barnich and C. Troessaert, Symmetries of Asymptotically Flat 4 Dimensional Spacetimes at Null Infinity Revisited, Phys. Rev. Lett. 105, 111103 (2010).

[53] A. Bagchi, Correspondence between Asymptotically Flat Spacetimes and Nonrelativistic Conformal Field Theories, Phys. Rev. Lett. 105, 171601 (2010).

[54] A. Bagchi, S. Detournay, and D. Grumiller, Flat-Space Chiral Gravity, Phys. Rev. Lett. 109, 151301 (2012).

[55] G. Barnich, Entropy of three-dimensional asymptotically flat cosmological solutions, J. High Energy Phys. 10 (2012) 095 .

[56] A. Bagchi, S. Detournay, R. Fareghbal, and J. Simon, Holography of 3d Flat Cosmological Horizons, Phys. Rev. Lett. 110, 141302 (2013).

[57] G. Barnich and C. Troessaert, Comments on holographic current algebras and asymptotically flat four dimensional spacetimes at null infinity, J. High Energy Phys. 11 (2013) 003.

[58] A. Bagchi, R. Basu, D. Grumiller, and M. Riegler, Entanglement Entropy in Galilean Conformal Field Theories and Flat Holography, Phys. Rev. Lett. 114, 111602 (2015).

[59] A. Bagchi, R. Basu, A. Kakkar, and A. Mehra, Flat holography: Aspects of the dual field theory, J. High Energy Phys. 12 (2016) 147.

[60] A. Bagchi, M. Gary, and Zodinmawia, Bondi-MetznerSachs bootstrap, Phys. Rev. D 96, 025007 (2017).

[61] H. Jiang, W. Song, and Q. Wen, Entanglement entropy in flat holography, J. High Energy Phys. 07 (2017) 142.
[62] L. Ciambelli, C. Marteau, A. C. Petkou, P. M. Petropoulos, and K. Siampos, Flat holography and Carrollian fluids, J. High Energy Phys. 07 (2018) 165.

[63] E. Hijano, Semi-classical $\mathrm{BMS}_{3}$ blocks and flat holography, J. High Energy Phys. 10 (2018) 044.

[64] R. Fareghbal and P. Karimi, Complexity growth in flat spacetimes, Phys. Rev. D 98, 046003 (2018).

[65] A. Ball, E. Himwich, S. A. Narayanan, S. Pasterski, and A. Strominger, Uplifting $\mathrm{AdS}_{3} / \mathrm{CFT}_{2}$ to flat space holography, J. High Energy Phys. 08 (2019) 168.

[66] E. Hijano, Flat space physics from AdS/CFT, J. High Energy Phys. 07 (2019) 132.

[67] D. Grumiller, P. Parekh, and M. Riegler, Local Quantum Energy Conditions in Non-Lorentz-Invariant Quantum Field Theories, Phys. Rev. Lett. 123, 121602 (2019).

[68] V. Godet and C. Marteau, Gravitation in flat spacetime from entanglement, J. High Energy Phys. 12 (2019) 057.

[69] R. Fareghbal and M. H. Shalamzari, First law of entanglement entropy in flat-space holography, Phys. Rev. D 100, 106006 (2019).

[70] T. Banks, Holographic space-time models in $1+1$ dimensions, arXiv:1506.05777.

[71] C. G. Callan, Jr., S. B. Giddings, J. A. Harvey, and A. Strominger, Evanescent black holes, Phys. Rev. D 45, R1005 (1992).

[72] D. Cangemi and R. Jackiw, Gauge Invariant Formulations of Lineal Gravity, Phys. Rev. Lett. 69, 233 (1992).

[73] The acronym BMS derives from seminal work by Bondi et al. and Sachs [74,75] on asymptotic symmetries of asymptotically flat spacetimes in four spacetime dimensions. In distinction to $\mathrm{BMS}_{4}$, the $\mathrm{BMS}_{2}$ supertranslations are radial. In Euclidean signature with Euclidean time $t \sim$ $t+\beta$ instead of Laurent modes Fourier modes are used, $L_{n}=\xi\left(\varepsilon=i e^{2 \pi i n t / \beta}, 0\right)$ and $M_{n}=\xi\left(0, \eta=e^{2 \pi i n t / \beta}\right)$, with no changes in the algebra.

[74] H. Bondi, M. van der Burg, and A. Metzner, Gravitational waves in general relativity VII. Waves from axi-symmetric isolated systems, Proc. R. Soc. A 269, 21 (1962).

[75] R. Sachs, Asymptotic symmetries in gravitational theory, Phys. Rev. 128, 2851 (1962).

[76] S. Detournay, T. Hartman, and D. M. Hofman, Warped conformal field theory, Phys. Rev. D 86, 124018 (2012).

[77] H. Afshar, S. Detournay, D. Grumiller, and B. Oblak, Nearhorizon geometry and warped conformal symmetry, J. High Energy Phys. 03 (2016) 187.

[78] H. Verlinde, Black holes and strings in two dimensions, in Trieste Spring School on Strings and Quantum Gravity (World Scientific, Singapore, 1991) pp. 178-207; the same lectures were given at MGVI in Japan, June, 1991.

[79] H. A. González, D. Grumiller, and J. Salzer, Towards a bulk description of higher spin SYK, J. High Energy Phys. 05 (2018) 083.

[80] See Supplemental Material at http://link.aps.org/ supplemental/10.1103/PhysRevD.101.086024 for more on the Maxwell algebra in $1+1$ dimensions and e.g. [90,91] as well as references therein for more on the Maxwell (super-) algebra in higher dimensions.

[81] S. Bonanos, J. Gomis, K. Kamimura, and J. Lukierski, Maxwell Superalgebra and Superparticle in Constant Gauge Backgrounds, Phys. Rev. Lett. 104, 090401 (2010). 
[82] J. Matulich, S. Prohazka, and J. Salzer, Limits of threedimensional gravity and metric kinematical Lie algebras in any dimension, J. High Energy Phys. 07 (2019) 118.

[83] Our BF theory has a linear Casimir, $Y$, and a bilinear Casimir, $C=X^{+} X^{-}-Y X$. Both are constant on shell and invariant under all boundary condition preserving transformations, $\delta_{\lambda} Y=0=\delta_{\lambda} C$. See [84,85] for more on $\mathrm{BF}$ theories, its deformation to Poisson-sigma models, and Casimirs appearing therein.

[84] N. Ikeda, Two-dimensional gravity and nonlinear gauge theory, Ann. Phys. (N.Y.) 235, 435 (1994).

[85] P. Schaller and T. Strobl, Poisson structure induced (topological) field theories, Mod. Phys. Lett. A 09, 3129 (1994).

[86] E. Witten, Coadjoint Orbits of the Virasoro Group, Commun. Math. Phys. 114, 1 (1988).

[87] A. Alekseev and S. L. Shatashvili, Path integral quantization of the coadjoint orbits of the Virasoro group and 2D gravity, Nucl. Phys. B323, 719 (1989).

[88] H. R. Afshar, Warped Schwarzian theory, J. High Energy Phys. 02 (2020) 126.
[89] J. Gegenberg, G. Kunstatter, and D. Louis-Martinez, Observables for two-dimensional black holes, Phys. Rev. D 51, 1781 (1995).

[90] T. M. Fiola, J. Preskill, A. Strominger, and S. P. Trivedi, Black hole thermodynamics and information loss in two- dimensions, Phys. Rev. D 50, 3987 (1994).

[91] S. Sachdev, Bekenstein-Hawking Entropy and Strange Metals, Phys. Rev. X 5, 041025 (2015).

[92] P. Chaturvedi, Y. Gu, W. Song, and B. Yu, A note on the complex SYK model and warped CFTs, J. High Energy Phys. 12 (2018) 101.

[93] Y. Gu, A. Kitaev, S. Sachdev, and G. Tarnopolsky, Notes on the complex Sachdev-Ye-Kitaev model, J. High Energy Phys. 02 (2020) 157.

[94] D. Grumiller, W. Kummer, and D. V. Vassilevich, Dilaton gravity in two dimensions, Phys. Rep. 369, 327 (2002).

[95] D. Grumiller and R. Meyer, Quantum dilaton gravity in two dimensions with fermionic matter, Classical Quantum Gravity 23, 6435 (2006).

[96] D. J. Gross, J. Kruthoff, A. Rolph, and E. Shaghoulian, $T \bar{T}$ in $\mathrm{AdS}_{2}$ and quantum mechanics, Phys. Rev. D 101, 026011 (2020). 